\title{
Donepezil Enhances Frontal Functional Connectivity in Alzheimer's Disease: A Pilot Study
}

\author{
Ludovica Griffanti $^{\mathrm{a}} \quad$ Gordon K. Wilcock $^{\mathrm{b}}$ Natalie Voets ${ }^{\mathrm{a}}$

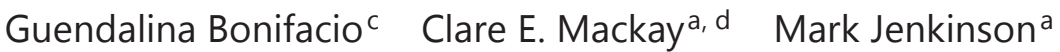 \\ Giovanna Zamboni ${ }^{a-c}$
}

${ }^{a}$ Centre for Functional Magnetic Resonance Imaging of the Brain (FMRIB), Nuffield

Department of Clinical Neuroscience, University of Oxford, ${ }^{b}$ Oxford Project to Investigate Memory and Ageing, Nuffield Department of Clinical Neuroscience, University of Oxford, Oxford, UK; ${ }^{C}$ Dipartimento di Scienze Biomediche, Metaboliche e Neuroscienze, Università di Modena e Reggio Emilia, Modena, Italy; ${ }^{d}$ Department of Psychiatry, University of Oxford, Oxford, UK

\section{Key Words}

Alzheimer's disease - Donepezil · Cholinesterase inhibitors · Functional magnetic resonance imaging $\cdot$ Resting-state networks · Functional connectivity

\section{Abstract}

Background: We have previously shown that increased resting-state functional magnetic resonance imaging (fMRI)-based functional connectivity (FC) within the frontal resting-state networks in Alzheimer's disease (AD) patients reflects residual, possibly compensatory, function. This suggests that symptomatic treatments should aim to enhance FC specifically in these networks. Methods: 18 patients with probable AD underwent brain MRI and neuropsychological assessment at baseline and after 12 weeks of treatment with donepezil. We tested if changes in cognitive performance after treatment correlated with changes in FC in restingstate networks known to be altered in AD. Results: We found increases in FC in the orbitofrontal network that correlated with cognitive improvement after treatment. The increased FC was greatest in patients who responded most to treatment. Conclusion: This 'proof of concept' study suggests that changes in network-specific FC might be a biomarker of pharmacological intervention efficacy in AD. 
Griffanti et al.: Donepezil Enhances Frontal Functional Connectivity in Alzheimer's Disease: A Pilot Study

\section{Introduction}

There is increasing evidence that neurodegenerative disorders including Alzheimer's disease (AD) reflect dysfunction of large-scale functional brain networks that can be studied with resting-state functional magnetic resonance imaging (rfMRI) [1]. Functional connectivity (FC) measures correlations of spontaneous fluctuations across brain regions, which can be clustered into these large-scale resting-state networks (RSNs) using recently developed independent component analysis (ICA) techniques. Several RSNs have been identified with these methods [2] and associated with specific cognitive functions [3]. It is anticipated that relatively simple measures of FC obtained with rfMRI, which does not suffer the complication of task-based fMRI, may serve in the future in clinical trials for testing the therapeutic effect of new treatments.

Recent ICA-based rfMRI studies including our own [4] showed that, among other RSNs, the default mode network (DMN), specifically engaged during self-reflection or when subjects are at rest, is disrupted in AD patients relative to healthy controls. Conversely, frontal and frontoparietal RSNs, which include brain regions associated with working memory and executive control, are enhanced in AD patients [5, 6]. By combining resting and task-related fMRI, we previously demonstrated that such increased FC in frontal RSNs in AD patients overlaps with task-specific hyperactivation that patients exhibit during successful performance of a memory task [4]. Based on this correspondence with task-specific fMRI, we argued that increased FC identified using rfMRI in these frontal networks reflects residual, possibly compensatory, cognitive ability in AD.

Our study raised an important issue: should an effective treatment decrease FC in patients or increase it in order to reflect a more efficient use of the remaining resources [5]? Based on our results, we argued that FC in frontal networks would be expected to increase in response to treatments aimed to enhance residual ability in AD patients [4]. Interestingly, cholinesterase inhibitor therapy, the only currently approved symptomatic treatment for mild-tomoderate $\mathrm{AD}$, has been shown to improve cognitive performance by upregulating frontal systems activation in previous task-based fMRI studies [7, 8]. However, the effect of treatment on ICA-based frontal networks reflecting residual cognitive ability in patients with AD has not been explored $[9,10]$.

In the present 'proof of concept' study, we aimed to test the effect of cholinesterase inhibitor therapy on the directionality of longitudinal changes in FC in these networks. We collected cognitive and multimodal MRI data from patients with AD at baseline (T0) and after 12 weeks of treatment with donepezil (T1). We then tested whether changes in cognition measured with the Montreal Cognitive Assessment (MoCA) correlated with longitudinal changes (increases or decreases) in FC. Based on our previous results, we hypothesized that cognitive improvement on cholinesterase inhibitor therapy would reflect increased FC specifically within frontal lobe RSNs previously shown to capture residual abilities in AD patients.

\section{Materials and Methods}

\section{Participants}

Eighteen patients (mean age $73.2 \pm 6.8$ years, female $/$ male $=8 / 10$ ) with a clinical diagnosis of probable AD [11] were recruited from the Oxford Project to Investigate Memory and Ageing (OPTIMA) and from the Oxford Memory Assessment Clinic at the John Radcliffe Hospital. Patients had never taken cholinesterase inhibitors. They were asked to participate with a carer or informant who knew them well enough to give information about their functioning and cognition. Diagnoses were given by an expert group that included a neurologist, 
Griffanti et al.: Donepezil Enhances Frontal Functional Connectivity in Alzheimer's Disease: A Pilot Study

a neuropsychologist, and 2 research nurses with expertise in dementia. Exclusion criteria were evidence or history of stroke, epilepsy, focal brain lesions, Parkinson's disease, frontotemporal, vascular or atypical dementia, or any major psychiatric disorder including major depression. They also included history of alcohol or substance abuse, contraindication to MRI, and active participation in a clinical drug trial. Participants suffering from hypercholesterolemia, diabetes, and hypertension were included in the study if they had been on stable medication for at least a year before the study enrolment. Details about these comorbidities and other risk factors are reported in table 1. Patients had not smoked, drunken alcohol, or taken caffeinated drinks for $24 \mathrm{~h}$ before the evaluations.

Patients underwent medical and neurological examination, neuropsychological assessment, and MRI scan at baseline (T0) and after 12 weeks (T1) of treatment with donepezil ( $5 \mathrm{mg}$ daily for the first 4 weeks, then $10 \mathrm{mg}$ daily). The prescription of cholinesterase inhibitors was independently given by the physician attending the memory clinic on the basis of clinical need and according to the NICE recommendation. The study was conducted under ethical approval from the Central Office for NHS Research Ethics Committees and the Bristol Frenchay Research Ethics Committee. All subjects gave written informed consent in accordance with the Declaration of Helsinki.

The neuropsychological assessment included: Mini-Mental State Examination (MMSE) [12], MoCA [13], Clinical Dementia Rating (CDR) Scale [14], Hopkins Verbal Learning TestRevised, backwards digits, category and letter fluency [15], and Geriatric Depression Scale. A semi-structured interview with the carer was carried out to gain information on the patient's cognitive status and included the CDR (informant part), Bristol Activities of Daily Living Scale Plus, and Neuropsychiatric Inventory with Caregiver Distress Scale [16]. Details about demographic, clinical, neuropsychological and anatomical characteristics are reported in table 1.

Differences $(\Delta)$ in the MoCA score were calculated $(\Delta=\mathrm{T} 1-\mathrm{T} 0)$ and used for correlation with rfMRI. The MoCA was specifically chosen as it is thought to be particularly sensitive to subtle cognitive changes by targeting frontal-executive functions in addition to memory and other cognitive domains $[13,17]$.

In addition, participants were classified according to their response to treatment as 'responders' $\left(\mathrm{n}=10\right.$, mean age 75.3 \pm 4.2 years, mean $\left.\mathrm{MMSE}_{\mathrm{T} 0}=21.7 \pm 3.6\right)$ or 'nonresponders' $\left(\mathrm{n}=8\right.$, mean age $70.5 \pm 8.6$ years, mean $\mathrm{MMSE}_{\mathrm{T} 0}=20.7 \pm 6.3$ ) (see table 1 for further details). They were considered 'responders' if an improvement was reported by the carer and demonstrated by at least two out of three tests of global cognition among MMSE $(\Delta>0)$, MoCA $(\Delta>$ $0)$, and $\operatorname{CDR}(\Delta<0)$.

\section{MRI Data Acquisition}

Scanning was performed at the Oxford Centre for Clinical Magnetic Resonance Research using a 3T Trio Siemens MRI scanner equipped with a 12-channel head coil. The neuroimaging protocol included: (1) rfMRI (EPI T2*-weighted images; TR/TE $=2,000 / 28$ ms; resolution = $3 \times 3 \times 3.5 \mathrm{~mm}^{3}$; FOV = $192 \mathrm{~mm}$; number of volumes = 180); (2) diffusion-weighted MRI (EPI T2*-weighted images; TR/TE $=3,000 / 28 \mathrm{~ms}$; resolution $=3 \times 3 \times 3 \mathrm{~mm}^{3}$; flip angle $89^{\circ} ; \mathrm{FOV}=224 \mathrm{~mm}$; number of diffusion directions $=12$; b-value $\left.=1,000\right)$, and ( 3 ) high-resolution T1-weighted 3D MP-RAGE images (TR/TE = 2,040/4.7 ms; resolution = $1 \times 1 \times 1 \mathrm{~mm}^{3}$; FOV $192 \mathrm{~mm}$ ). Baseline MRI data are also included in a previously published cross-sectional comparison with controls and patients with mild cognitive impairment [4].

\section{Structural MRI Analyses}

Prior to the rfMRI analyses, we tested on T1- and diffusion-weighted MRI data if significant structural changes had occurred in the 12-week interval to rule out that differences in FC were driven by longitudinal changes in gray and/or white matter. T1-weighted images 
Dementia

Cognitive Disorders

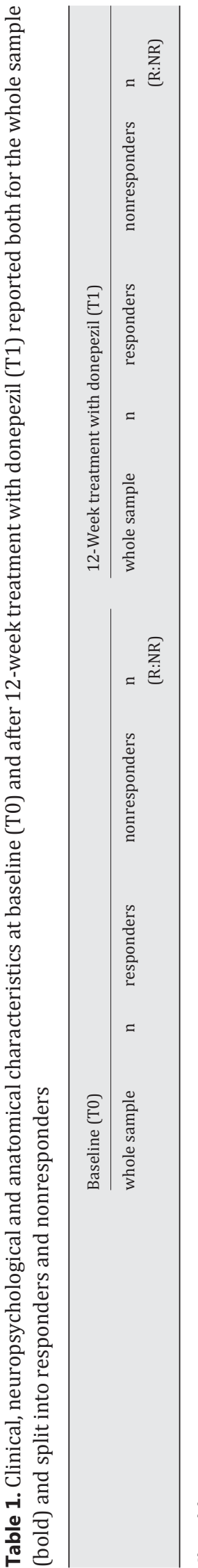

Dement Geriatr Cogn Disord Extra 2016:6:518-528

\begin{tabular}{l|l} 
DOI: $10.1159 / 000450546$ & (c) 2016 The Author(s). Published by S. Karger AG, Basel
\end{tabular} www.karger.com/dee

Griffanti et al.: Donepezil Enhances Frontal Functional Connectivity in Alzheimer's Disease: A Pilot Study

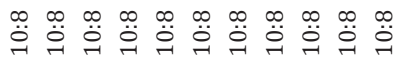

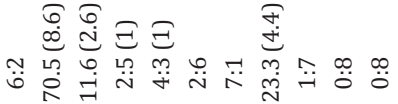

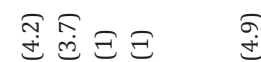

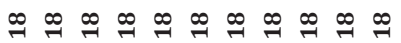

包官 范 N

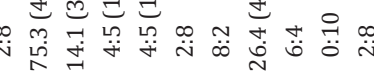

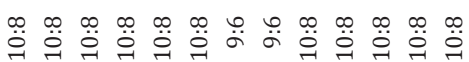

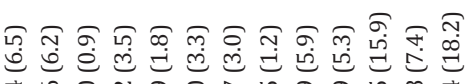

苟

ง

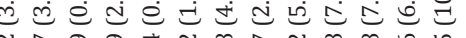

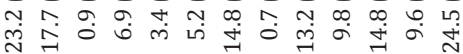

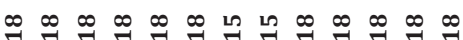

F⿱一兀)

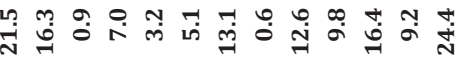

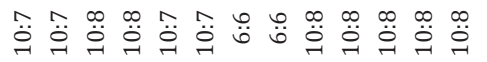

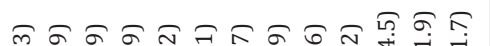
అ

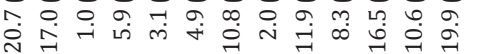

อ

m

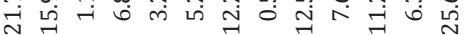

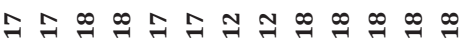

K56

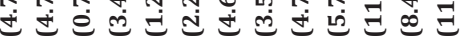
กิ

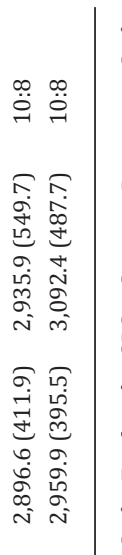

$\stackrel{\infty}{\rightarrow} \stackrel{\infty}{\rightarrow}$

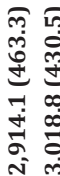

ले

$\stackrel{\infty}{\stackrel{\infty}{0}} \stackrel{\oplus}{\dot{0}}$

$\rightarrow$ 章

๑า

$\therefore$ ก

a. a

我

กิ

กิ

웡

눙요

ले ने

$\stackrel{\infty}{\sim}$

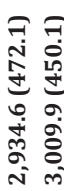

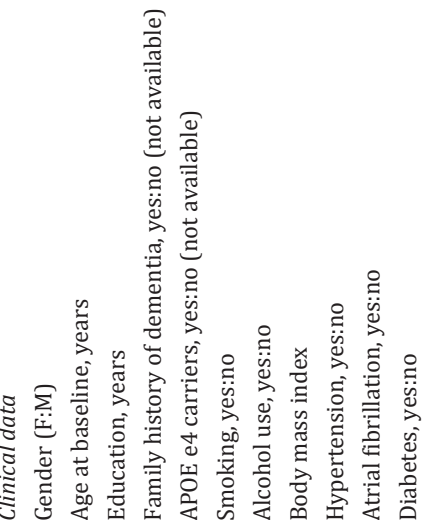




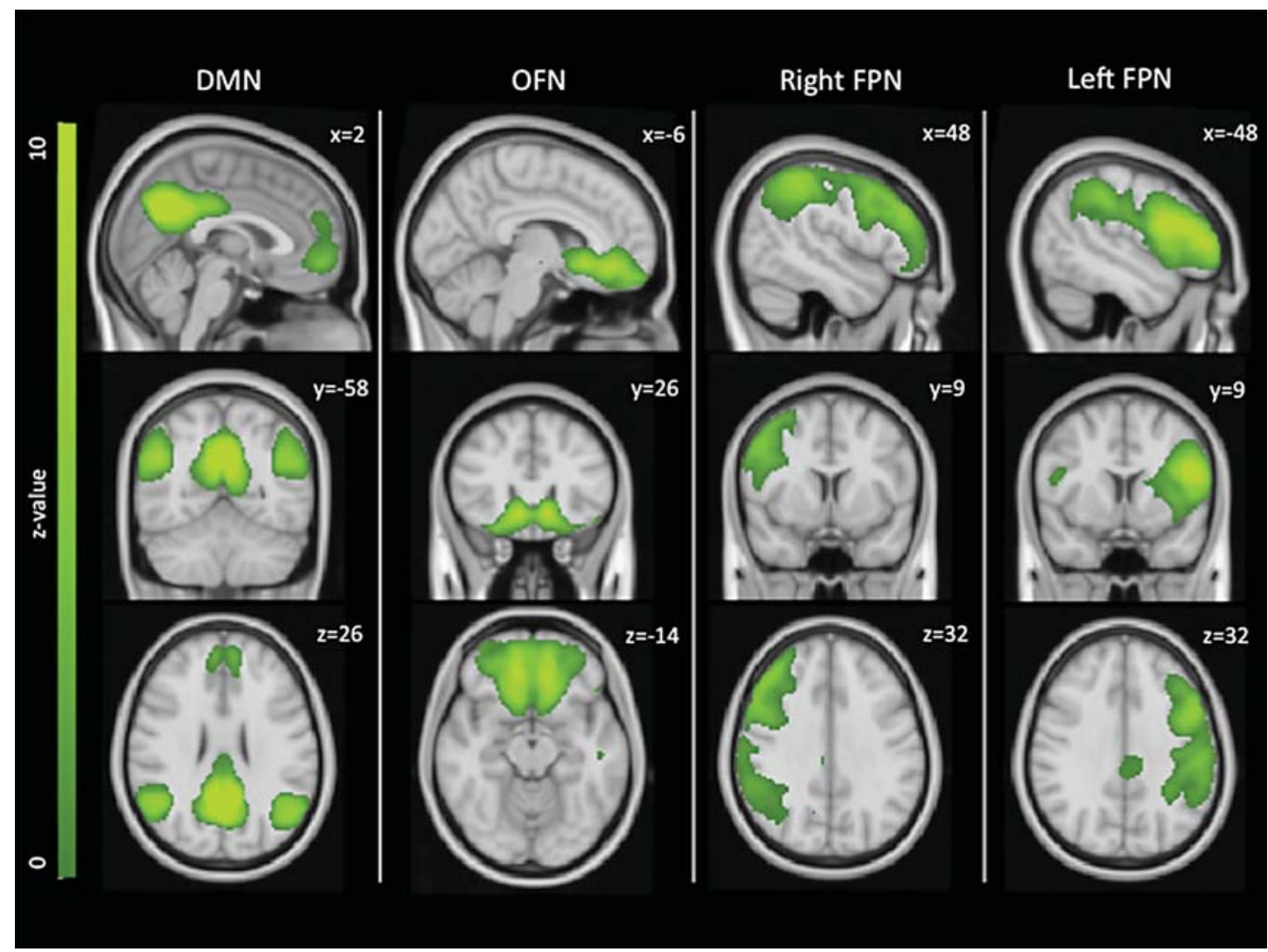

Fig. 1. RSNs of interest. The DMN, OFN and left and right frontoparietal networks (FPN) were the focus of the current study, as they were previously found to be significantly altered in AD patients relative to controls [4]. Images are shown in radiological convention and MNI coordinates are reported.

were analyzed with FSL-VBM, an optimized voxel-based morphometry (VBM) protocol [18, 19], to evaluate longitudinal changes in gray matter volume. Diffusion-weighted images were corrected for head motion and eddy currents, then FA images were created by fitting a tensor model to the raw diffusion data using FDT, and they were brain-extracted using BET. Voxelwise statistical analysis of the FA data was carried out using Tract-Based Spatial Statistics (TBSS) [20] to evaluate longitudinal changes in white matter integrity.

\section{rfMRI Analysis}

rfMRI data preprocessing with FSL [21] included brain extraction, head motion correction, unwarping using field maps, spatial smoothing (FWHM $6 \mathrm{~mm}$ ), high-pass filtering (150 s), physiological denoising using physiological noise modelling [22, 23], and registration of EPI images to high-resolution T1 with boundary-based registration [24] and to MNI space using linear and nonlinear registration. Dual regression [25] was used to derive subject-specific versions of the 25 RSN maps from our previous study [4]. Briefly, in the first step of dual regression, the template including the 25 components maps was used in a general linear model fit (as spatial regressors) against the fMRI preprocessed data, the output being the corresponding temporal dynamics for each component and subject. Secondly, these time courses were used in a second general linear model (as temporal regressors) against the fMRI data to estimate subject-specific spatial maps. As a result, the values in these maps (parameter estimates, PE) represent the connectivity of each voxel with the main regions of the specific 
Fig. 2. Areas showing significant positive correlation between changes in MoCA score and changes in FC (red-yellow) and those showing a significant FC increase after treatment (paired t test, blue-light blue) with the orbitofrontal RSN. Images are shown in radiological convention and $\mathrm{MNI}$ coordinates are reported.
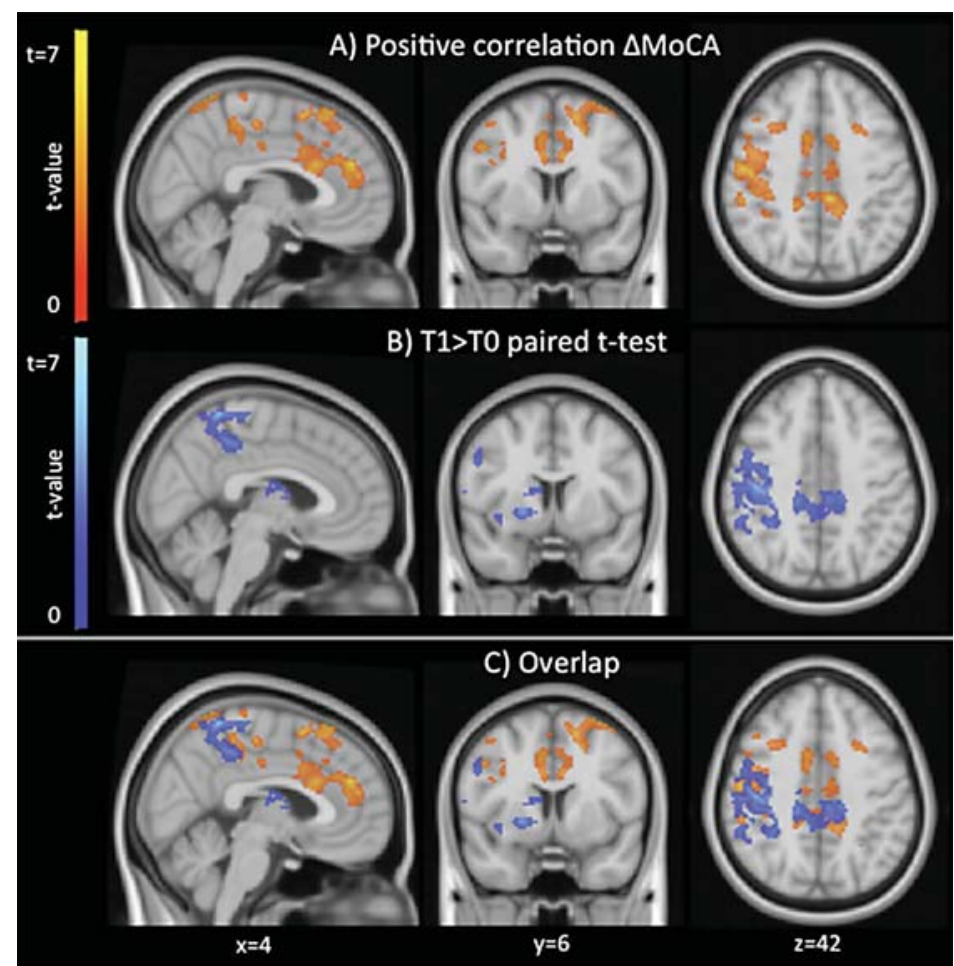

RSN. For subsequent statistical analyses, we focused on the 4 RSNs that we had previously found to be significantly altered in AD patients relative to controls [DMN, orbitofrontal network (OFN), left and right frontoparietal RSNs; fig. 1].

First, we performed paired t tests on these 4 RSNs to obtain longitudinal FC changes between T0 and T1 $(\triangle \mathrm{FC})$. Because we did not have a placebo control group (it would have been unethical to deprive patients from a treatment already approved for $\mathrm{AD}$ ), we reasoned that a change in FC could plausibly be interpreted as an effect of treatment only if it correlated with a positive change $(\Delta>0)$ in cognitive scores. Therefore, we performed correlational analyses to test whether $\triangle \mathrm{FC}$ correlated with $\triangle \mathrm{MoCA}$. Results were considered significant for $\mathrm{p}<0.05$, cluster-corrected for multiple comparisons after initial cluster thresholding at $\mathrm{p}_{\text {uncorr }}<0.05$.

Secondly, for those networks showing a significant correlation with cognitive improvement, we performed two explicatory analyses: (a) a paired t test to explore the general pattern of longitudinal changes between $\mathrm{T} 0$ and $\mathrm{T} 1$ (without restricting to regions of significant correlation with cognitive scores); (b) a time-by-group analysis comparing responders and nonresponders at the two time points to test if one group contributed more than the other to FC changes ( $p<0.05$, cluster-corrected for multiple comparisons after initial cluster thresholding at $\mathrm{p}_{\text {uncorr }}<0.05$ ).

\section{Results}

Paired t test analyses showed no significant differences in neuropsychological and anatomical characteristics between baseline (T0) and after 12 weeks of treatment (T1). There were no significant differences between responders and nonresponders in demographic, 
Fig. 3. PEs extracted from the regions showing a significantly increased FC after treatment in the subjects for which the MoCA score was available (17 subjects), classified in responders (red, solid lines) and nonresponders (black, dashed lines).
Griffanti et al.: Donepezil Enhances Frontal Functional Connectivity in Alzheimer's Disease: A Pilot Study

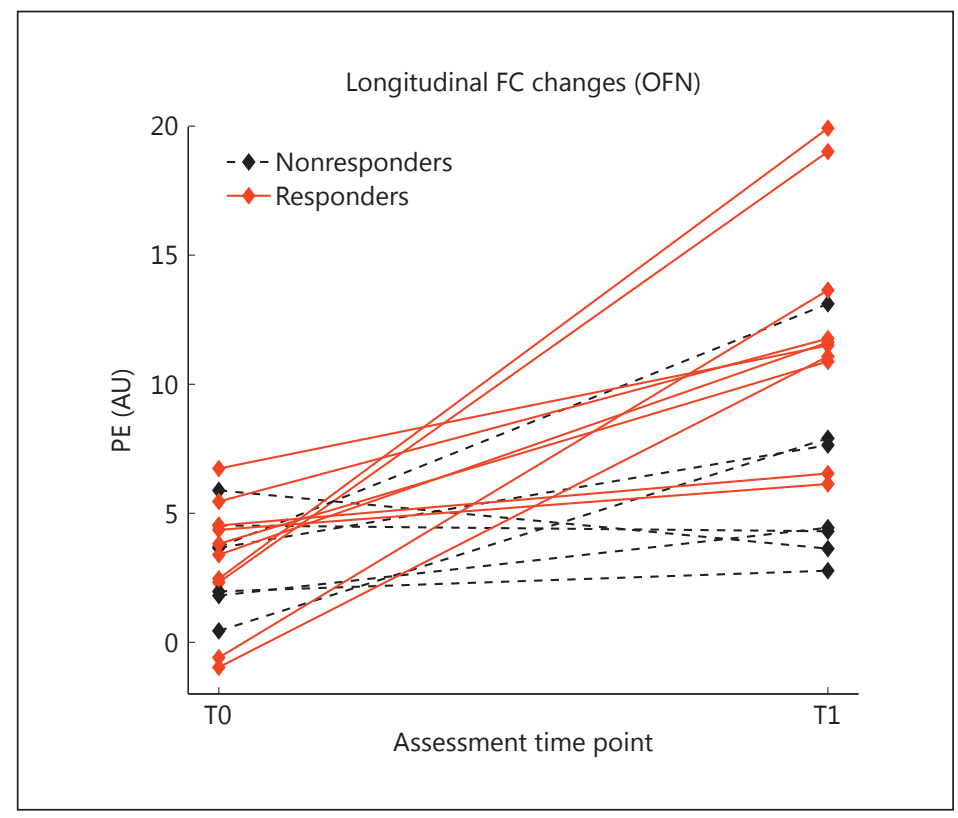

clinical, neuropsychological and anatomical characteristics at baseline. We observed no differences in these metrics at $\mathrm{T} 1$, except the expected significant difference in $\triangle \mathrm{MMSE}$ (responders: $1.5 \pm 1.5$; nonresponders $-1.7 \pm 3.4 ; \mathrm{p}=0.004$ ) and $\triangle$ MoCA (responders: $1.8 \pm$ 2.6; nonresponders $-1.3 \pm 1.1 ; \mathrm{p}=0.01$ ).

No significant structural changes between T0 and T1 were observed with VBM [18] (p < 0.05 , cluster-corrected for multiple comparisons after initial cluster thresholding at $\mathrm{p}_{\text {uncorr }}<$ $0.05)$ and TBSS [20] ( $<<0.05$, corrected for multiple comparisons with threshold-free cluster enhancement) analyses, ruling out that any changes in FC are driven by significant longitudinal structural change.

Analysis of rfMRI data showed that the OFN was the only network showing significant results in both correlational and paired t test analyses $\left(\mathrm{p}_{\text {corr }}<0.05\right)$. There was a significant positive correlation between increased FC within the OFN and increased MoCA scores in the medial prefrontal cortex within the anterior cingulate gyrus, precuneus/posterior cingulate gyrus, and right dorsolateral frontal regions (fig. 2a, c, red-yellow). Direct comparison of FC between T0 and T1 in the OFN performed with paired t test explicatory analysis (fig. 2b, c, bluelight blue) also showed increased FC in the precuneus/posterior cingulate gyrus and right dorsolateral frontal regions, in addition to subcortical regions in close proximity with the basal forebrain cholinergic system (sublenticular forebrain, anterior insula, and caudate). No significant FC decreases were observed. By repeating the analyses adding voxel-based measures of gray matter, the results did not change in localization but were less significant ( $\mathrm{p}_{\text {uncorr }}<0.01$ ).

Within the areas showing a significantly increased FC after treatment (fig. 2b, blue-light blue), we extracted the mean PE from the subject-specific spatial maps. The PE values, shown in figure 3 for each subject at T0 and T1, represent the average connectivity of those areas with the main regions of the network. The subjects are classified as responders (red) and nonresponders (black), showing a greater increase in FC for the responders (although not statistically significant).

The voxel-wise explicatory time-by-group analysis comparing responders and nonresponders revealed a time-by-group interaction (increased FC higher in responders relative to nonresponders) significant at the trend level $\left(\mathrm{p}_{\text {corr }}=0.053\right)$. This interaction was driven by 
Fig. 4. Results of time-by-group explicatory analysis. Comparison between $\mathrm{T} 0$ and $\mathrm{T} 1$ within the responders group: areas showing a significant FC increase with the OFN are shown in red. Images are shown in radiological convention and MNI coordinates are reported.

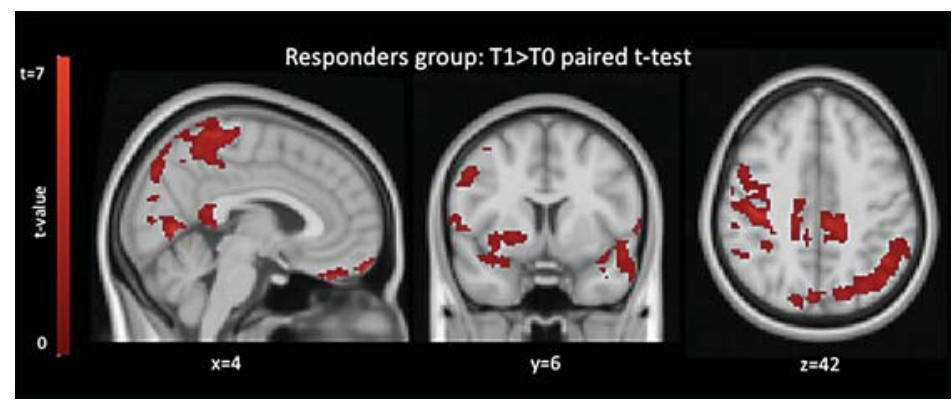

a significant increase $\left(\mathrm{p}_{\text {corr }}<0.05\right)$ in $\mathrm{FC}$ within the responders group between T0 and T1 (fig. 4) in medial and lateral parietal regions including the precuneus, bilateral dorsal and orbitofrontal regions, and right anterior insula and putamen. There were no significant longitudinal changes in the nonresponders group. FC in the OFN was not statistically different at baseline between responders and nonresponders but only at T1.

\section{Discussion}

We aimed to test the effect of currently approved treatment for $\mathrm{AD}$ on the directionality of FC changes measured with ICA-based rfMRI. Cross-sectional studies have shown that FC within frontal RSNs is increased in patients with AD relative to controls [5, 6]. Here we present a longitudinal pilot study on patients treated with cholinesterase inhibitor therapy (donepezil). We found that cognitive improvement following 12 weeks of treatment positively correlated with the magnitude of longitudinal increases of FC only within the OFN. This suggests that treatment enhances residual function subsided by frontal networks.

The areas of significant correlation between $\triangle \mathrm{FC}$ within the OFN and $\triangle \mathrm{MoCA}$ scores were localized in the medial cortex (anterior cingulate gyrus and precuneus/posterior cingulate gyrus) and dorsolateral frontal cortex. These areas all receive cholinergic projections from the basal forebrain cholinergic system $[9,26]$ and have previously been shown to correlate volumetrically with the basal forebrain cholinergic system in patients [27]. Li et al. [9] also observed a significant increase in regional cerebral blood flow and FC in the neural substrates of the medial cholinergic pathway (middle and posterior cingulate cortex) in mild AD subjects after donepezil treatment, and the changes were correlated with changes in cognitive performance.

These results indicate a network-specific rather than global, nonspecific effect of donepezil on FC. Interestingly, among the RSNs of interest, the OFN is the only one that included the basal forebrain cholinergic system [28], suggesting that the OFN is specifically sensitive to cholinergic-enhancing treatment. This finding of specific modulation of frontal lobe regions is consistent with previous PET and fMRI studies [29]. Increased glucose metabolism was observed with FDG PET in patients who responded to treatment with cholinesterase inhibitors [30, 31]. In particular, Potkin et al. [30] found a marked metabolic increase in the prefrontal system, predominantly on the right side, which is in line with the pattern found in our study. In fact, although the clusters surviving cluster correction for multiple comparisons are clearly right-sided, the uncorrected maps also included most of the corresponding areas in the left hemisphere. Task-based fMRI studies on cholinesterase inhibitors showed increased brain activation during working memory tasks [29], although these results may have been biased by the selection of specific fMRI tasks to activate particular brain regions. Instead, our results show an effect of donepezil that is task-independent and network-specific. 
The paired t test analysis performed to directly compare baseline (T0) and 12-week posttreatment (T1) showed a significant increase in FC within the OFN in the precuneus and right dorsolateral frontal regions (also emerged from the correlational analyses), but also in subcortical regions including anterior insula, putamen and caudate. This confirms our hypothesis that increased FC, observed previously in frontal networks in AD [5, 6], is further enhanced by cholinergic treatment. The overlap of the results of the paired t test analysis with the results of the correlational analysis suggests that the increase in FC is an effect of treatment, not a nonspecific effect of disease progression, which, on the contrary, has been shown to be associated with decreases in FC over time in a previous ICA-based rfMRI study on the natural history of AD [6]. Thus, the increase in FC that we detected most likely represents an effect of the treatment, which affected cortical and subcortical areas of the cholinergic system. Interestingly, it was the increased FC of the more cortical areas (medial and dorsofrontal) that directly correlated with cognitive improvement, as no subcortical regions resulted from the correlational analysis. The results of the time-by-group analysis, although underpowered for the small number of subjects, further showed that the correlation was driven by increased FC in responders rather than decreases in nonresponders, although there were no significant differences in demographic, clinical, neuropsychological and anatomical characteristics between the two groups (table 1). Careful inspection of the outliers revealed that the nonresponder patient with the highest FC increase was one of the youngest subjects (63 years old) and had the lowest MMSE ( 10 both at T0 and at T1), and that the responder with the least FC increase had borderline cognitive features suggesting a possible diagnosis of posterior cortical atrophy. This suggests that different clinical phenotypes have different effects of FC and should be further investigated, and possibly controlled for, in future larger studies. Future studies should also investigate if differences between responders and nonresponders are specifically driven by pharmacokinetic features such as differences in CYP2D6 status [32].

One limitation of this study is the small sample size. However, this was a 'proof of concept', whose results encourage further research in this direction. Another limitation of this study is that the diagnosis was given on clinical basis (according to McKhann et al. [11]) and not further supported by biomarkers of amyloidosis. We also did not have a placebo control group of the study, as it would have been unethical to delay a treatment already approved for $\mathrm{AD}$, but future studies could, for example, selectively recruit for this category AD patients for which this therapy is contraindicated.

\section{Conclusion}

Our pilot study shows that $\mathrm{FC}$ increases in the OFN reflect the positive effect of treatment with donepezil, and possibly other cholinesterase inhibitors, in AD patients. Although additional work is needed to validate the use of rfMRI in clinical trials, our results suggest that it has the potential to be used as surrogate outcome measure to test the effect of new treatments, and may have the greatest utility in early studies to detect an efficacy signal over relatively short time frames.

\section{Acknowledgments}

We thank all the participants and their families for their commitment and support. We wish to acknowledge all OPTIMA staff and in particular research assistant Erin Drazich and research nurse Ellen McCullogh for help in performing neuropsychological assessment and acquiring MRI data; OCMR staff and in particular research assistant Steve Knight for help 
Griffanti et al.: Donepezil Enhances Frontal Functional Connectivity in Alzheimer's

Disease: A Pilot Study

during imaging acquisition; the FMRIB analysis group and in particular Steve Smith for help with independent component analysis of resting data. This study was supported by the Oxford NIHR Biomedical Research Centre (to L.G., G.W, C.E.M. and G.Z.) and by Sir Stewart Halley Trust (to G.Z.).

\section{Disclosure Statement}

The authors declare that the research was conducted in the absence of any commercial or financial relationships that could be construed as a potential conflict of interest.

\section{References}

$>1$ Pievani M, de Haan W, Wu T, Seeley WW, Frisoni GB: Functional network disruption in the degenerative dementias. Lancet Neurol 2011;10:829-843.

-2 De Luca M, Beckmann CF, De Stefano N, Matthews PM, Smith SM: fMRI resting state networks define distinct modes of long-distance interactions in the human brain. Neuroimage 2006;29:1359-1367.

-3 Smith SM, Fox PT, Miller KL, Glahn DC, Fox PM, Mackay CE, Filippini N, Watkins KE, Toro R, Laird AR, Beckmann CF: Correspondence of the brain's functional architecture during activation and rest. Proc Natl Acad Sci USA 2009;106:13040-13045.

4 Zamboni G, Wilcock GK, Douaud G, Drazich E, McCulloch E, Filippini N, Tracey I, Brooks JC, Smith SM, Jenkinson M, Mackay CE: Resting functional connectivity reveals residual functional activity in Alzheimer's disease. Biol Psychiatry 2013;74:375-383.

5 Johnson KA, Sperling RA, Sepulcre J: Functional connectivity in Alzheimer's disease: measurement and meaning. Biol Psychiatry 2013;74:318-319.

6 Damoiseaux JS, Prater KE, Miller BL, Greicius MD: Functional connectivity tracks clinical deterioration in Alzheimer's disease. Neurobiol Aging 2012;33:828.e19-e30.

-7 Saykin AJ, Wishart HA, Rabin LA, Flashman LA, McHugh TL, Mamourian AC, Santulli RB: Cholinergic enhancement of frontal lobe activity in mild cognitive impairment. Brain 2004;127:1574-1583.

-8 Petrella JR, Prince SE, Krishnan S, Husn H, Kelley L, Doraiswamy PM: Effects of donepezil on cortical activation in mild cognitive impairment: a pilot double-blind placebo-controlled trial using functional MR imaging. AJNR Am J Neuroradiol 2009;30:411-416.

>9 Li W, Antuono PG, Xie C, Chen G, Jones JL, Ward BD, Franczak MB, Goveas JS, Li SJ: Changes in regional cerebral blood flow and functional connectivity in the cholinergic pathway associated with cognitive performance in subjects with mild Alzheimer's disease after 12-week donepezil treatment. Neuroimage 2012;60:1083-1091.

10 Sole-Padulles C, Bartres-Faz D, Llado A, Bosch B, Pena-Gomez C, Castellvi M, Rami L, Bargallo N, Sanchez-Valle R, Molinuevo JL: Donepezil treatment stabilizes functional connectivity during resting state and brain activity during memory encoding in Alzheimer's disease. J Clin Psychopharmacol 2013;33:199-205.

11 McKhann GM, Knopman DS, Chertkow H, Hyman BT, Jack CR Jr, Kawas CH, Klunk WE, Koroshetz WJ, Manly JJ, Mayeux R, Mohs RC, Morris JC, Rossor MN, Scheltens P, Carrillo MC, Thies B, Weintraub S, Phelps CH: The diagnosis of dementia due to Alzheimer's disease: recommendations from the National Institute on AgingAlzheimer's Association workgroups on diagnostic guidelines for Alzheimer's disease. Alzheimers Dement 2011;7:263-269.

12 Folstein MF, Folstein SE, McHugh PR: 'Mini-mental state'. A practical method for grading the cognitive state of patients for the clinician. J Psychiatr Res 1975;12:189-198.

13 Nasreddine ZS, Phillips NA, Bedirian V, Charbonneau S, Whitehead V, Collin I, Cummings JL, Chertkow H: The Montreal Cognitive Assessment, MoCA: a brief screening tool for mild cognitive impairment. J Am Geriatr Soc 2005;53:695-699.

14 Morris JC: The Clinical Dementia Rating (CDR): current version and scoring rules. Neurology 1993;43:2412-2414.

15 De Jager CA, Hogervorst E, Combrinck M, Budge MM: Sensitivity and specificity of neuropsychological tests for mild cognitive impairment, vascular cognitive impairment and Alzheimer's disease. Psychol Med 2003;33: 1039-1050.

$\$ 16$ Cummings JL: The neuropsychiatric inventory: assessing psychopathology in dementia patients. Neurology 1997;48:S10-S16.

17 Markwick A, Zamboni G, de Jager CA: Profiles of cognitive subtest impairment in the Montreal Cognitive Assessment (MoCA) in a research cohort with normal Mini-Mental State Examination (MMSE) scores. J Clin Exp Neuropsychol 2012;34:750-757.

18 Douaud G, Smith S, Jenkinson M, Behrens T, Johansen-Berg H, Vickers J, James S, Voets N, Watkins K, Matthews PM, James A: Anatomically related grey and white matter abnormalities in adolescent-onset schizophrenia. Brain 2007;130:2375-2386. 
Good CD, Johnsrude IS, Ashburner J, Henson RN, Friston KJ, Frackowiak RS: A voxel-based morphometric study of ageing in 465 normal adult human brains. Neuroimage 2001;14:21-36.

-20 Smith SM, Jenkinson M, Johansen-Berg H, Rueckert D, Nichols TE, Mackay CE, Watkins KE, Ciccarelli O, Cader MZ, Matthews PM, Behrens TE: Tract-based spatial statistics: voxelwise analysis of multi-subject diffusion data. Neuroimage 2006;31:1487-1505. Jenkinson M, Beckmann CF, Behrens TE, Woolrich MW, Smith SM: FSL. Neuroimage 2012;62:782-790. Harvey AK, Pattinson KT, Brooks JC, Mayhew SD, Jenkinson M, Wise RG: Brainstem functional magnetic resonance imaging: disentangling signal from physiological noise. J Magn Reson Imaging 2008;28:1337-1344.

-23 Brooks JC, Beckmann CF, Miller KL, Wise RG, Porro CA, Tracey I, Jenkinson M: Physiological noise modelling for spinal functional magnetic resonance imaging studies. Neuroimage 2008;39:680-692.

24 Greve DN, Fischl B: Accurate and robust brain image alignment using boundary-based registration. Neuroimage 2009;48:63-72.

25 Filippini N, MacIntosh BJ, Hough MG, Goodwin GM, Frisoni GB, Smith SM, Matthews PM, Beckmann CF, Mackay CE: Distinct patterns of brain activity in young carriers of the APOE-epsilon4 allele. Proc Natl Acad Sci USA 2009;106:7209-7214.

26 Selden NR, Gitelman DR, Salamon-Murayama N, Parrish TB, Mesulam MM: Trajectories of cholinergic pathways within the cerebral hemispheres of the human brain. Brain 1998;121(Pt 12):2249-2257.

27 Teipel SJ, Flatz WH, Heinsen H, Bokde AL, Schoenberg SO, Stockel S, Dietrich O, Reiser MF, Moller HJ, Hampel H: Measurement of basal forebrain atrophy in Alzheimer's disease using MRI. Brain 2005;128:2626-2644.

-28 Hedreen JC, Struble RG, Whitehouse PJ, Price DL: Topography of the magnocellular basal forebrain system in human brain. J Neuropathol Exp Neurol 1984;43:1-21.

29 Venneri A: Imaging treatment effects in Alzheimer's disease. Magn Reson Imaging 2007;25:953-968.

-30 Potkin SG, Anand R, Fleming K, Alva G, Keator D, Carreon D, Messina J, Wu JC, Hartman R, Fallon JH: Brain metabolic and clinical effects of rivastigmine in Alzheimer's disease. Int J Neuropsychopharmacol 2001;4: 223-230.

31 Tune L, Tiseo PJ, Ieni J, Perdomo C, Pratt RD, Votaw JR, Jewart RD, Hoffman JM: Donepezil HCl (E2020) maintains functional brain activity in patients with Alzheimer disease: results of a 24-week, double-blind, placebocontrolled study. Am J Geriatr Psychiatry 2003;11:169-177.

-32 Seripa D, Bizzarro A, Pilotto A, D’Onofrio G, Vecchione G, Gallo AP, Cascavilla L, Paris F, Grandone E, Mecocci P, Santini SA, Masullo C, Pilotto A: Role of cytochrome P4502D6 functional polymorphisms in the efficacy of donepezil in patients with Alzheimer's disease. Pharmacogenet Genomics 2011;21:225-230. 